\title{
Procedure Start Day
}

National Cancer Institute

\section{Source}

National Cancer Institute. Procedure Start Day. NCI Thesaurus. Code C117531.

The first day of the procedure. 